\title{
Level Set Method for Tip Shape Evolution Simulation for Atom Probe Tomography
} Jie Bao ${ }^{1}$, Zhijie Xu², Robert Colby ${ }^{3}$, Suntharampillai Thevuthasan ${ }^{4}$, Arun Devaraj ${ }^{3}$

${ }^{1}$ Nuclear Science Division, Pacific Northwest National Laboratory, Richland, WA, USA;

${ }^{2}$ Computational Mathematics Division, Pacific Northwest National Laboratory, Richland, WA, USA;

${ }^{3}$ Environmental Molecular sciences Laboratory, Pacific Northwest National Laboratory, Richland, WA, USA;

${ }^{4}$ Qatar Energy and Environmental Research Institute, Qatar, UAE

Invented in $1967^{1}$, atom probe tomography (APT) was designed to visualize chemical heterogeneity present in a small volume of a specimen with a sub-nanometer spatial resolution ${ }^{2-5}$. Numerical simulation plays an important role in studying the sample geometry evolution and corresponding electrical field changes during field evaporation of needle shaped specimens in APT. The level set method can provide sub-grid accuracy on tracking the solid-vacuum interface and much higher computational efficiency than other models that require an atomic level grid. The modeling details are introduced in our previous work ${ }^{6}$. The proposed approach is applied to predict the tip evaporation of the specimen, which is (100) chromium (Cr) single crystalline thin film grown on a (100) single crystalline magnesium oxide (MgO) substrate. Through comparison with experimental observation, the level set method shows the capability of providing atomic level accuracy while using a relatively coarse simulation grid that is about 5 times the atomic cell. This leads to a huge advantage in computational efficiency. The scanning transmission electron microscopy (STEM) image of the tip geometry before and after evaporation is shown in Fig. 1(a), and the comparison between STEM image and simulation results is shown in Fig.1(b).

Additionally level set simulations were used to investigate the dynamic tip shape evolution of oxide multilayer materials. Due to their interesting interfacial properties oxide multilayered structures have acquired a lot of attention in several scientific and industrial fields, such as magnetic storage media and microelectronics. The dynamic tip shape evolution of a composite material consisting of alternating layers of $\mathrm{CeO}_{2}$ and $\mathrm{ZrO}_{2}$ in two orientations (topdown and side-ways) was studied by the numerical simulations. In order to study how the sample geometry and electrical field changes affect the species density distribution on the detector, a trajectory simulation model by Runge-Kutta method is integrated into the level set solid-vacuum interface tracking model. Because the grid size of the level set method is around 5 times the atomic volume, a local grid refinement method is used for initialization of the starting positions of evaporated atoms for the trajectory calculation. Fig. 2 shows the simulation results for the $\mathrm{CeO}_{2}$ and $\mathrm{ZrO}_{2}$ horizontal multilayer sample evaporation. Fig. 2(a) and (b) show the tip geometry and electric field evolution during evaporation after 0 and 5000 atoms are evaporated respectively. Fig. 2(c) shows the end position of atoms flying over the counter electrode, and Fig. 2(d) shows the atom density distribution on the selected region as shown on Fig. 2(c). Because the evaporation strength of $\mathrm{ZrO}_{2}$ is about double that of $\mathrm{CeO}_{2}, \mathrm{CeO}_{2}$ is evaporated much faster and easier than $\mathrm{ZrO}_{2}$, which causes concaves for $\mathrm{CeO}_{2}$ on the tip surface. The concaves on the tip surface lead to higher density of electrical field lines, and make the trajectories of Ce closer to each other. This is consistent with the narrower band of $\mathrm{Ce}$ and wider band of $\mathrm{Zr}$ in the density distribution as shown in Fig. 2(d), which is fairly well matched to the experiment observations and measurements. Similarly, Fig. 3 shows the simulation results for the $\mathrm{CeO}_{2}$ and $\mathrm{ZrO}_{2}$ vertical multilayer sample evaporation.

Reference

1. Müller, E. W.; Panitz, J. A.; McLane, S. B., The atom-probe field ion microscope. Preview of Scientific Instruments 1968, 39 (1), 83-86. 
2. Vurpillot, F.; Bostel, A.; Blavette, D., Trajectory overlaps and local magnification in threedimensional atom probe. Applied Physics Letters 2000, 76 (21), 3127-3129.

3. Cerezo, A.; Godfrey, T. J.; Smith, G. D. W., Application of a position - sensitive detector to atom probe microanalysis. Review of Scientific Instruments 1988, 59 (6), 862-866.

4. Blavette, D.; Bostel, A.; Sarrau, J. M.; Deconihout, B.; Menand, A., An atom probe for threedimensional tomography. Nature 1993, 363, 432-435.

5. Blavette, D.; Déconihout, B.; Chambreland, S.; Bostel, A., Three-dimensional imaging of chemical order with the tomographic atom-probe. Ultramicroscopy 1998, 70 (3), 115-124.

6. Xu, Z.; Li, D.; Xu, W.; Devaraj, A.; Colby, R.; Thevuthasan, S., Simulation of Heterogeneous atom probe tip shapes evoltion during field evaporation using a level set method and different evaporation models. Computer Physics Communications 2015, In press.

7. This work was funded as a part of laboratory directed research and development as a part of chemical imaging initiative in pacific northwest national laboratory. RC acknowledge funding from EMSL distingusihed Wiley Postdoctoral Fellowship.

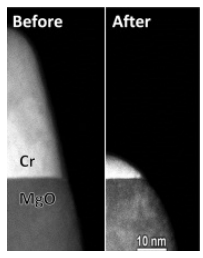

(a)

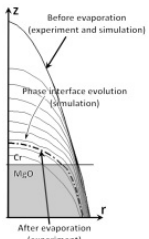

(b)

Fig. 1: (a) scanning transmission electron microscopy (STEM) side view of the tested specimen before and after evaporation, (b) Comparison between the simulation results and the observation of the experiment.
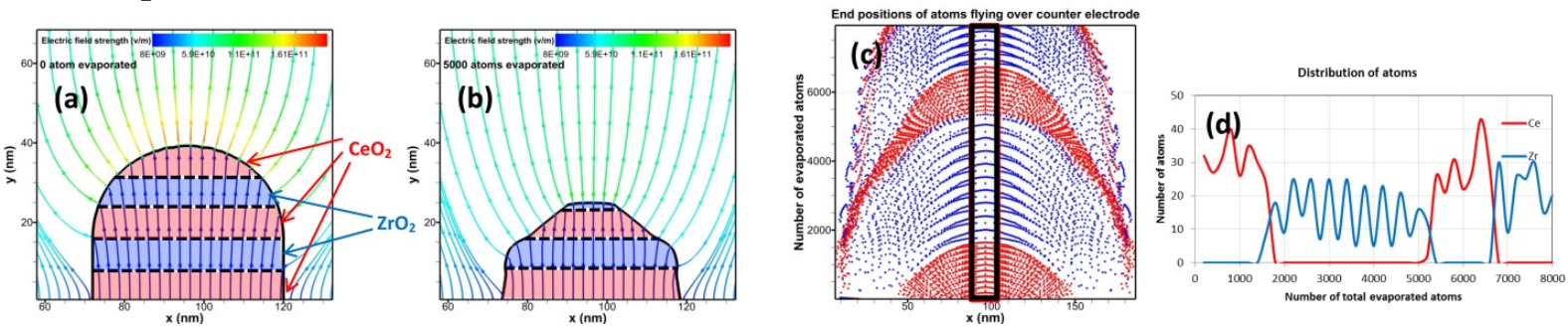

Fig. 2: Simulation results for the $\mathrm{CeO}_{2}$ and $\mathrm{ZrO}_{2}$ horizontal multilayer sample evaporation; (a) and (b): the tip geometry and electric field evolution during evaporation after 0 and 5000 atoms are evaporated respectively; (c): end position of atoms flying over counter electrode; (d): atoms density distribution on the selected region as shown on (c).
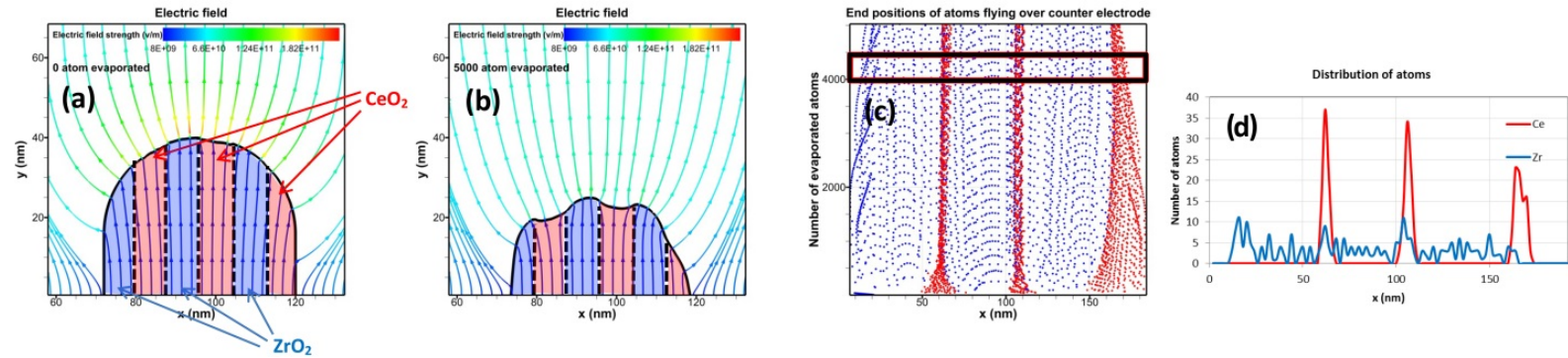

Fig. 3: Simulation results for the $\mathrm{CeO}_{2}$ and $\mathrm{ZrO}_{2}$ vertical multilayer sample evaporation; (a) and (b): the tip geometry and electric field evolution during evaporation after 0 and 5000 atoms are evaporated respectively; (c): end position of atoms flying over counter electrode; (d): atoms density distribution on the selected region as shown on (c). 